\title{
Fibroma cementificante apical: um diagnóstico diferencial
} Apical cementifing fibroma: diferencial diagnosis

\author{
Denise Tostes Oliveira * \\ Alberto Consolaro** \\ Iris Malagoni Marques*** \\ Tadashi Akatsu**** \\ Pantelis Varvaki Rados*****
}

OLIVEIRA, D.T. et alii. Fibroma Cementificante apical: um diagnóstico diferencial.

OLIVEIRA, D.T. et alii. Apical cementifying fibroma: a differential diagnosis.

\section{Resumo:}

Um caso de fibroma cementificante na regiăo apical de um incisivo central superior com vitalidade, em uma paciente negra de 52 anos de idade, é apresentado. Os seus aspectos clínicos, radiográficos e microscópicos såo discutidos sob o ponto de vista do diagnóstico diferencial entre os cementomas e as lesões periapicais inflamatórias.

\section{Summary:}

A case of cementifying fibroma at the apex of a vital upper left central incisor occurred in a 52 year-old black woman, is presented. The clinical, radiographic and microscopic features are discussed, by the point of view of the differential diagnosis between the cementomas and the inflammatory periapical lesions.

\section{UNITERMOS:}

Cementoma - Tumores Odontogênicos - Lesões fibro-ósseas dos maxilares.

\section{Introdução}

fibroma cementificante é um tumor odontogênico benigno do grupo dos cementomas ${ }^{1}$. A lesão têm origem no ligamento periodontal2,3,4 de dentes com vitalidade pulpar, sendo inicialmente detectada através de exames radiográficos de rotina. O crescimento é lento, assintomático, mas pode provocar ex- pansão óssea. Os aspectos radiográficos variam de acordo com o estágio de desenvolvimento do tumor as características microscópicas são muito semelhantes em muitos casos as do fibroma ossificante ${ }^{5}$. Ele ocorre predominantemente na mandíbula, no sexo feminino, com prevalência maior na raça negra quando comparada com outras amostragens da populaçăo ${ }^{2,3}$.
* Pós-graduanda em Patologia Bucal da Faculdade de Odontologia de Bauru - USP.

** Professor Doutor do Departamento de Patologia da Faculdade de Odontologia de Bauru - USP.

*** Professora de Cirurgia Bucal da Universidade Federal de Uberlândia.

*** Professor de Patologia da Universidade Estadual de Londrina.

***** Professor de Patologia Eucal da UFRGS e PUCRS.

R. Fac. Odontol.

Porto Alegre

v. 34

N. 1

p.9-12

SETEMBRO

1993 


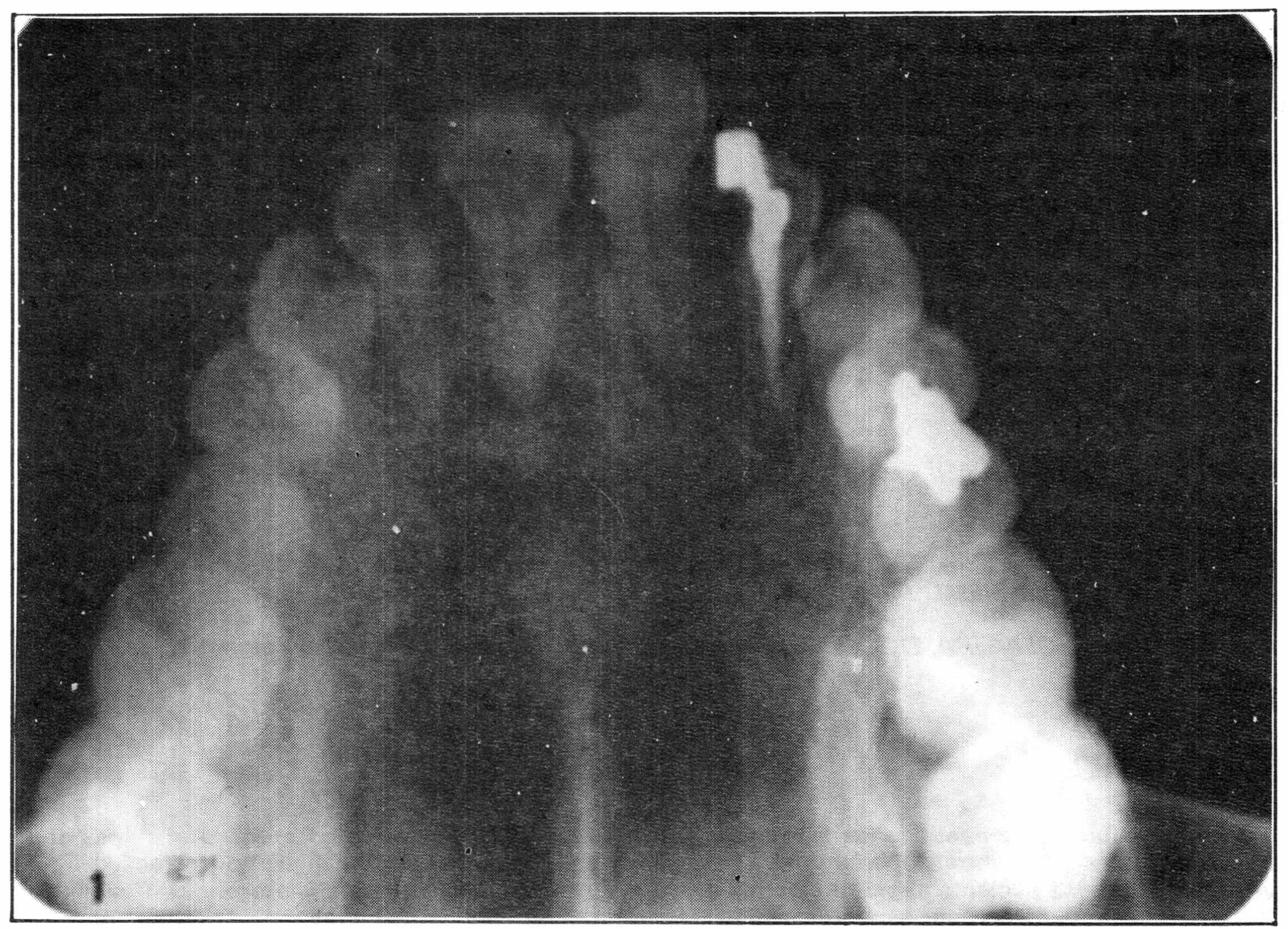

\section{Relato do Caso}

aciente do sexo feminino, de 52 anos, raça negra, apresentouse no Serviço de Triagem da Univerśidade Federal de Uberlândia para exame estomatológico de rotina no qual detectou-se, radiograficamente, uma lesão periapical no incisivo central superior esquerdo.

No exame clínico intra-bucal observou-se condições de normalidade nos tecidos moles, doença periodontal comum nos dentes superiores, além de trauma oclusal no incisivo central superior direito que apresentava-se com mobilidade intensa. O dente com a alteração periapical tinha uma coloração normal, vitalidade pulpar e ausência de sensibilidade dolorosa à percussão. Radiograficamente, a lesão era circunscrita, de contornos nítidos, com $1 \mathrm{~cm}$ de diâmetro, radiolúcida, mas com áreas radiopacas focais e difusas na região central, sem relação com o ápice dentário (figura 1). O diagnóstico presuntivo estabelecido foi de fibroma cementificante.

$\mathrm{O}$ paciente foi submetido a tratamento periodontal no qual houve exodontia do incisivo central superior direito. Para a lesão apical detectada, a conduta adotada foi o tratamento endodôntico, apicetomia e curetagem apical do incisivo central superior esquerdo. Com o levantamento do retalho cirúrgico, uma pequena expansão da tábua óssea vestibular foi notada na região correspondente ao periápice afetado. Após a cirurgia, o controle radiográfico mantido não apresentou sinais de recidiva.

A peça cirúrgica era constituída por fragmentos de tecido mole com partes menores de tecido duro, medindo em conjunto $0,8 \times 0,7 \mathrm{~cm}$ nos seus maiores eixos. Microscopicamente a lesão era formada por um tecido conjuntivo fibroso ricamente celularizado, cujos fibroblastos jovens distribuiam-se ora organizadamente, ora ao acaso, apresentando ainda áreas focais de tecido cementóide mineralizado as vezes de aspecto regular, outras vezes de aspecto irregular, provavelmente em função da curetagem promovida nos procedimentos cirúrgicos. Não havia infiltrado inflamatório nos tecidos observados. 0 diagnóstico estabelecido foi fibroma cementificante. 


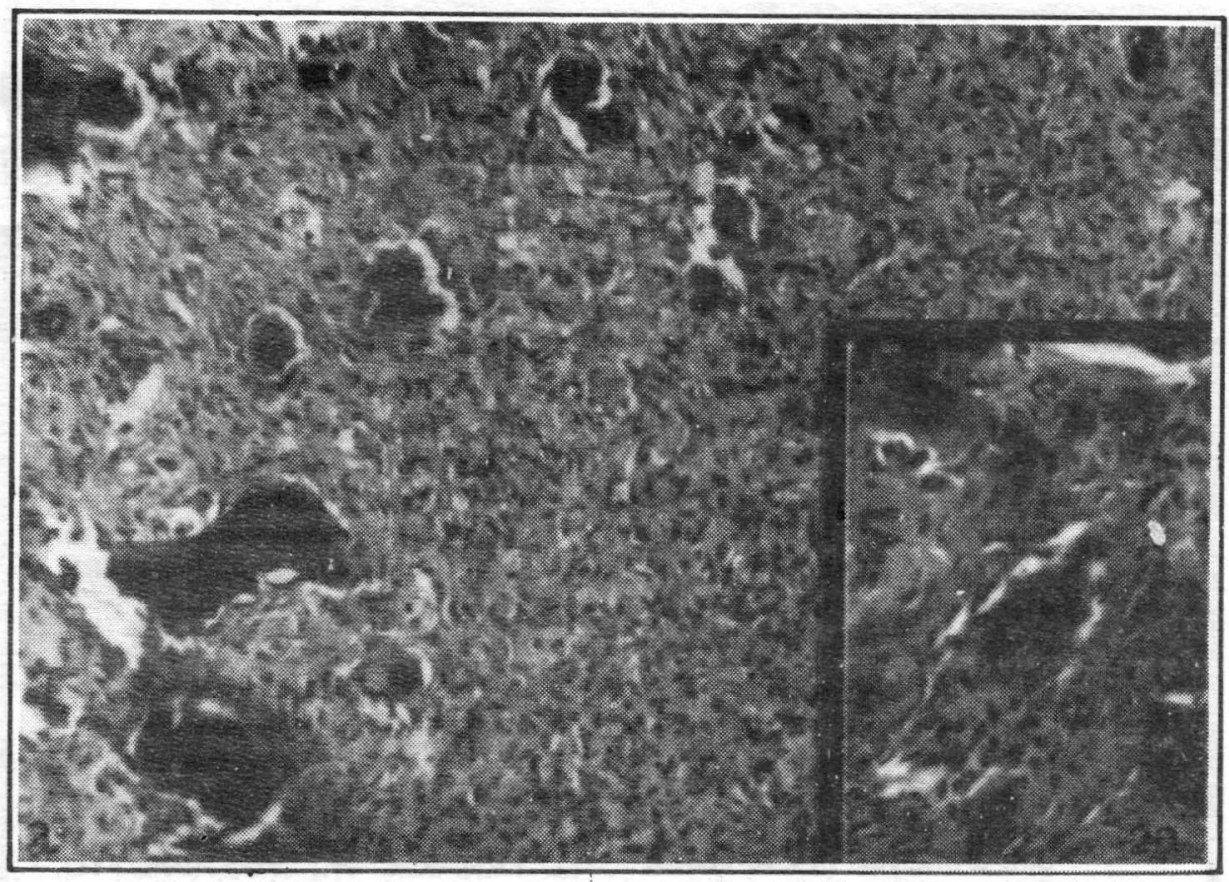

\section{Discussåo}

ligamento periodontal contém células blasticas que sob condiçð̃es patológicas produzem tumores compostos de cemento, osso lamelar e tecido conjuntivo, ou ainda, uma combinação dos três tipos teciduais de origem mesenquimal ${ }^{3}$. Os cementomas, a partir de 1961, foram classificados pela $\mathrm{WHO}^{6}$ (World' Health Organization) em quatro tipos: displasia cementária periapical, fibroma cementificante, cementoblastoma benigno e cementoma gigantiforme. Embora existam características peculiares a cada um deles, o diagnóstico diferencial pode depender de uma associação entre os achados clínicos, radiográficos e histopatológicos ${ }^{2}$. Alem dessa associaçăo ser importante na determinaçăo do diagnóstico definitivo, ela se faz também importante no diagnóstico diferencial dos cementomas com as outras lesర̃es fibro-osseas dos maxilares, haja vista a impossibilidade de distinguir um tecido osteóide de cementóide na microscopia de luz, mesmo utilizando-se de técnicas especiais de colorações e histoquímica.

Os aspectos radiográficos dos cementomas apresentam variações de acordo com os seus diferentes estágios de maturação. Inicialmente osteolítico, radiolúcido, e constituído por tecido conjuntivo fibroso com áreas de diferenciação cementoblástica. cuja matriz produzida mineraliza-se gradativamente. Assim, com o tempo, há o aparecimento de áreas focais radiopacas (ver figura 1) que aumentam em número e tamanho, e mais tardiamente preenchem toda a lesão, exceto a região periférica, mantendo-se um halo radiolúci do, separando-o do osso vizinho ${ }^{7}$. Este halo se faz importante no diagnóstico diferencial com os osteomas e osteítes condensantes ${ }^{7}$. A relação destas áreas radiopacas é um detalhe radiográfico e diagnóstico importante, pois sua continuidade com o ápice radicular, inclusive com perda dos seus contornos, se faz presente no cementoblastoma benigno 8,9 . Na displasia cementária periapical, ainda que os contornos anatômicos sejam visualizados, a continuidade entre o ápice dentário e as áreas radiopacas quase sempre existe, pelo menos em alguns pon$\operatorname{tos}^{10}$. Por outro lado, no fibroma cementificante, esta continuidade em geral não está presente. As massas radiopacas são mais centrais e isoladas, livres em relação as estruturas dentárias relacionadas, a não ser nos estágios finais de esclerose total da lesão ${ }^{7}$. Tal aspecto radiografico pode ser observado na figura 1.

A localização é um dado importante no diagnóstico diferencial dos cementomas. É sabido que as displasias cementárias aplicais envolvem de forma múltipla os incisivos inferiores, especialmente as mulheres negras ao redor de 30 anos de idade $4,10,11$, ainda que nos japone ses ocorrem mais na região dos prémolares e caninos inferiores ${ }^{12}$. O cementoblastoma benigno, por sua vez, preferencialmente ocorre na regiåo apical dos molares, em particular, no primeiro molar inferior ${ }^{8,9} \mathrm{e}$, tal como todos os cementomas, é mais freqüente nos indivíduos da raça negra em idade jovem ${ }^{3,4}$. Ainda que não tenhamos dados estatísticos de grandes séries de fibroma cementificante, em função de sua origem e dos vários casos isolados relatados na literatura $13,14,15,16$, ele pode ocorrer em qualquer parte dos maxilares, (sem predominância marcante para um determinado local), como ocorre com os outros cementomas citados. Na região anterior da maxila, tal como ocorreu em nosso caso, os cementomas, entretanto são raros. HAMNER et alii ${ }^{3}$ (1968) descreveu que $79 \%$ dos casos ocorrem na mandíbula, sendo a região dos prémolares a área mais afetada de ambos os maxilares, 80 e $82 \%$ para mandíbula e maxila respectivamente.

Assim sendo, associando-se o fator racial, a localização, as imagens radiográficas, além dos aspectos clínicos tal como a presença de vitalidade pulpar do dente afetado, o diagnóstico presuntivo da lesão foi de fibroma cementificante, posteriormente confirmado pela análise microscópica da lesão. No caso apresentado a lesåo já possuia os focos radiopacos característicos, no entanto, em estágios anteriores, a mesma provavelmente simulava com certeza uma lesão apical de natureza inflamatória, e caso houvesse superposição de fatores locais como
F. Fac. Odontol. v. 34
N. 1 p. -12 
cárie dentária e mesmo necrose pulpar, o diagnóstico diferencial seria dificultado. Sendo assim é importante ressaltar que no diagnóstico de lesões apicais, inflamatórias cuja origem, aparentemente, é de difícil explicação, deve-se incluir os cementomas, especialmente o fibroma cementificante como uma das hipóteses de diagnóstico.

\section{Referências Bibliográficas}

1. GORLIN, R.J. Odontogenic tumors. In: GORLIN, R.J. \& GOLDMAN, H.M., eds. Thoma's oral pathology. 6th ed. St. Louis, Mosby, 1970. p. 481-515.

2. HALL, E.H.; NAULOR, G.D.; MOHR, R.W.; WARNOCK, R.R. Early agressive cemento-ossifying fibroma: a disgnosic and treatment dilema. Oral Surg., 63: 132-6, 1987.

3. HAMNER, J.E.; SCOFIELD, H. H; CORNYIN, J. Benign fibro-osseous jaw lesions of periodontal membrane origin: a analysis of 249 cases. Cancer, 22: 861-78, 1968.

4. ZEGARELLI, E.; KUTSCHER, A. H.; NAPOLI, N.; IURONO, F.; HOFFMAN, P. The cementoma: a study of $230 \mathrm{pa}-$ tients with 435 cementomas. Oral Surg., 17(2): 219-24, 1964.

5. STRADER, R.J. Mature neoplastic cementifying fibroma: report a case. J. oral Surg., 29: 277-9, 1971.

6. PINDBORG, J.J. \& KRAMER, J R. Tipos histológicos de tumores odontogenicos, quistes de los maxilares y lesiones afines. Genebra, RotoSadag, 1975. p. 20.

7. WOOD, N.K. \& GOAZ, P.W. Diagnóstico diferencial das lesðos bucais. $2^{\mathrm{a}}$ ed. Rio de Janeiro, Guanabara Koogan, 1983, p. 293-6, 4436.

8. CHERRICK, H.M.; KING, O.H.; LUCATORTO, F.M. and SUGGS, D.M. Benign cementoblastoma: a clinicopathologic evaluation. Oral Surg., 37(1): 54-63, 1974.
9. PAPAGEORGE, M.B.; CATALDO, E.; NGHIEM, F.T. Cementoblastoma involving multiple deciduous teeth. Oral Surg., 63: 602-5, 1987.

10. CHAUDRY, A.P.; SPINK, J.H. and GORLIN, R.J. Periapical fibrous dysplasia (cementoma). J. oral Surg., 16: 4838, 1958.

11. BADEN, E.; SAROFF, S.A. Periapical cemental dysplasia and periodontal disease: a case report with review of the literature. J. Periodont., 58(3): 187-91, 1987.

12. TANAKA, H.; YOSHIMOTTO, A.; TOYAMA, Y; TAKASHI, I.; HAYASAKA, N.; MORO, I. Periapical cemental dysplasia with multiple lesions. Int. J. Oral Maxillofac. Surg. 16: 757-63, 1987.

13. ALBRIGHT, J.E.; TERRY, B.C.; BAKER, R.D. Cementifying fibroma of the mandible. Report of case. J. Oral Surg., 32: 294-9, 1974.

14. KUROYANAGI. K.; KAWABATA, T.; MIYACHI, S.; EDA, S. Cementifying fibroma: re port of three cases. Bull Tokyo dent. Coll., 14(4): 195-204, 1973.

15. PEDERSEN, G.W. Fibro-osseous lesion of the mandible: cementifying fibroma. Report of case. J. ora Surg., 29: 280-4, 1971.

16. SUGIMURA, M.; OKUNAGA, T.; YONEDA, T.; SHIBA, R.; AOBA, T.; YOSHIOKA, C.; OKADA, $Y$. Cementifying fibroma of the maxilla. Report of a case. Int. J. oral Surg., 10: 298-303, 1981. 